\title{
Effect of IPMSM d-q Axis Parameters According to Magnetic Saturation and Armature Reaction
}

\begin{abstract}
Kibong Jang ${ }^{\dagger}$
Abstract - FEA was used to analyze inductance and torque of IPMSM. Torque and inductance are analyzed on the dq-axis. It was shown that Ld and Lq have harmonic components, and magnitude as well as phase of the harmonics varies according to the current values. This paper shows the relationship between these inductance harmonics and the 6th harmonic component of torque.
\end{abstract}

Keywords: IPM, Harmonics, Magnetic saturation, Vector control, Inductance, Flux linkage

\section{Introduction}

Interior permanent magnet (IPM) motors can use various control methods because reluctance torque is utilized by field oriented control (FOC), but accurate parameters, such as the flux linkage of the permanent magnets and the difference between the $\mathrm{d}$ - and q-axis inductance, are required. Therefore, precise inductance measurements and calculations have been researched for many years [1-2]. However, salient motors, such as IPM motors, have nonlinear parameter characteristics generally, so there is also ongoing research which models theses nonlinearities and applies these models to calculate the torque [3-4]. Some papers show that a modified coordinate transformation is used to control the torque because of the nonlinear characteristics [5]. This method improves the torque calculation after modifying the transformation matrix, but it is difficult to consider the complicated inductance variation due to the saturation phenomena.

However, it is very rare to consider the parameter nonlinearities when utilizing FOC even though some research has been done. That is, the torque, $\mathrm{T}_{\mathrm{e}}$, uses equation 1 . In this equation, the parameters, $\mathrm{L}_{\mathrm{d}}$ and $\mathrm{L}_{\mathrm{q}}$, are assumed to be constants, and the PM flux linkage, $\psi_{\mathrm{f}}$, is also assumed to be a constant generally. Thereby, the torque is calculated to be constant for constant $i_{d}$ and $i_{q}$ currents.

$$
\begin{aligned}
T_{e} & =\frac{m P}{2}\left[\Psi_{d} i_{q}-\Psi_{q} i_{d}\right] \\
& =\frac{m P}{2}\left[\Psi_{f} i_{q}-\left(L_{d}-L_{q}\right) i_{q} i_{d}\right]
\end{aligned}
$$

On the other hand, the inductances and flux linkage are not linear in real cases shown in Fig. 1, which shows that the torques have ripple. Fig. 2 depicts the torque FFT

\footnotetext{
$\dagger$ Corresponding Author: Dept. of Electrical Engineering, Changwon

National University, Korea. (kbjang@changwon.ac.kr)

Received: July 9, 2014; Accepted: September 29, 2014
}

Table.1. Specification of IPM motor

\begin{tabular}{c|c}
\hline Specifications & Value \\
\hline Rated voltage / Current & $12[\mathrm{~V}] / 30[\mathrm{~A}]$ \\
\hline Rated torque / Speed & $0.8[\mathrm{Nm}] / 3,000[\mathrm{rpm}]$ \\
\hline Air gap / Br & $1[\mathrm{~mm}] / 1.2[\mathrm{~T}]$ \\
\hline Outer / Inner / Stack length & $60 / 28 / 50[\mathrm{~mm}]$ \\
\hline Poles / Slots & $4 / 6$ \\
\hline $\mathrm{R}_{\mathrm{s}} / \mathrm{L}_{\mathrm{d}} / \mathrm{L}_{\mathrm{q}}$ & $0.04[\Omega] / 0.18 / 0.31[\mathrm{mH}]$ \\
\hline
\end{tabular}

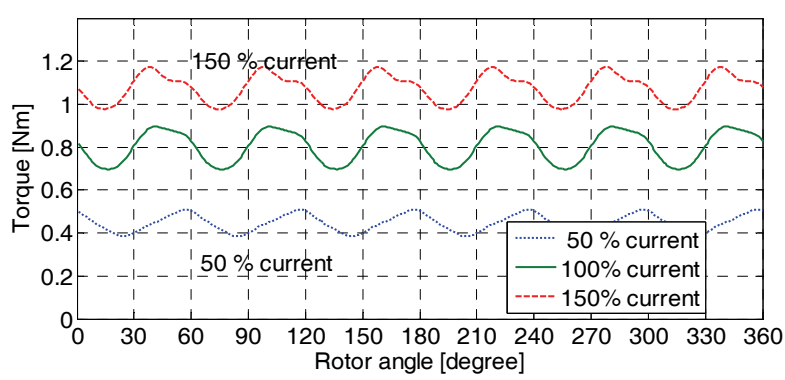

Fig. 1. Torque under the loads

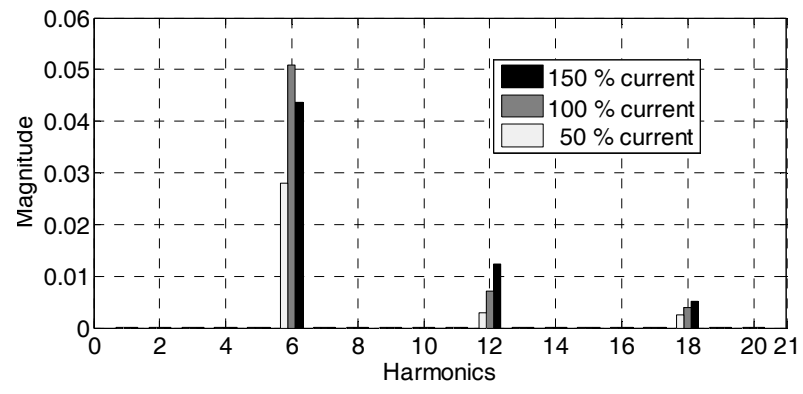

Fig. 2. Torque FFT Analysis

analysis, which has high $6^{\text {th }}$ harmonic components in the torques due to the nonlinear material and motor structure.

This paper presents the flux linkage and inductance variations though the FEA results of the IPM Motor in Table 1, and the effect in the $\mathrm{d}-\mathrm{q}$ axis and the relationship with the torque are studied. 


\section{Flux linkage Calculation}

\subsection{Flux linkage calculation in stationary coordinate}

As shown in equation 1 , the torque is proportional to the amplitude of the magnet flux linkage, so the accurate calculation of flux linkage is very important. Conventionally, the flux linkage value used is the average value of the magnet flux linkage without applying the phase currents to the windings. However, the applied currents induce core saturation and the amplitude of the magnet flux linkage will be different for real IPM motors. IPM motors have more a complicated shape for the flux linkage because of the saliency. FEA can analyze the flux distribution more precisely.

There are two steps necessary to calculate the flux linkage value of only the permanent magnets when applying the phase currents.

$1^{\text {st }}$ step: While applying the phase currents, the permeability of the each element from the calculated results needs to be stored after performing the nonlinear analysis.

$2^{\text {nd }}$ step: After changing the current to $0[\mathrm{~A}]$ from the state of the first step and making the source of the flux only from the permanent magnets, the flux linkage is reanalyzed and calculated.

Fig. 3 shows the A-phase flux linkage caused by the rated current powered on the d-axis, and the graphs show total flux linkage of the permanent magnets and current, flux linkage of only the current, excluding the impact of the permanent magnets, and flux linkage of only the permanent magnets. The d-axis current implies the direction which

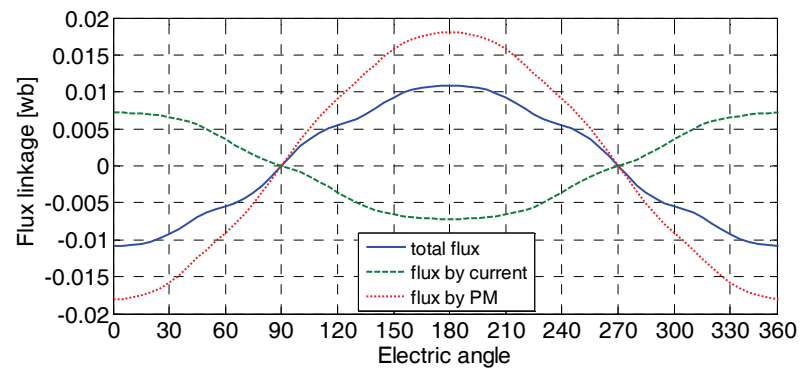

Fig. 3. Flux linkages of d-axis current

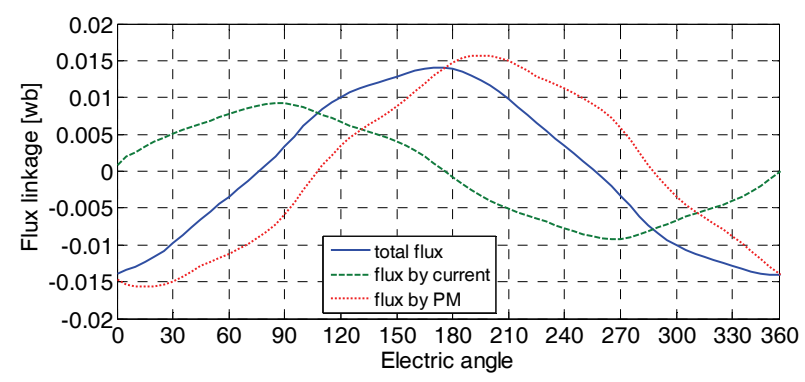

Fig. 4. Flux linkages of q-axis current

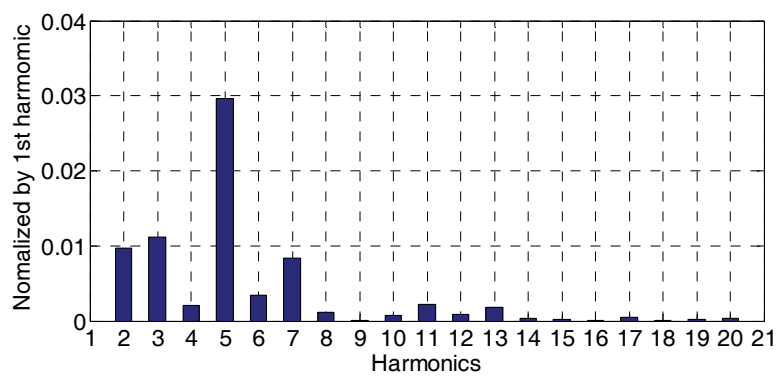

Fig. 5. q-axis flux linkage FFT analysis

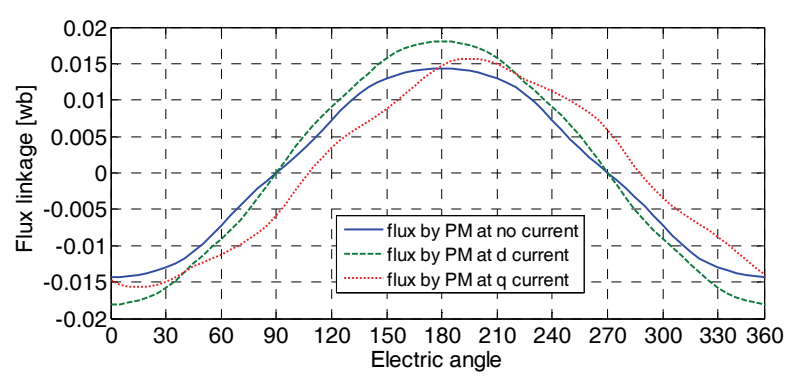

Fig. 6. Flux linkages of the magnets

reduces the flux linkage of the permanent magnets. The total flux linkage decrease, as shown in Fig. 3, and the current includes harmonic components causing partial iron saturation, but the flux linkage is symmetric.

Fig. 4 shows the A-phase flux linkage when applying the q-axis current. There is 90 degree phase difference between the flux linkages of the q-axis current and of the permanent magnets. Moreover, the armature reaction causes asymmetric saturation, which gives rise to the asymmetry of the flux linkage, which is not sinusoidal and contains harmonic components. The 5th harmonic component is relatively large as indicated in Fig. 5.

Fig. 6 shows flux linkage caused by only the permanent magnets when the rated current flows through both $\mathrm{d}$ axis and q-axis. The graphs represent flux linkage of the permanent magnets at no current, flux linkage of the permanent magnets when the d-axis current flows, and flux linkage of the permanent magnets when the q-axis current flows.

Compared to the flux linkage at no load current (that is, when the current is at $0 \mathrm{~A}$ ), the pattern of the flux linkage of the permanent magnets with the q-axis current has harmonics and is asymmetric because of the saturation. Moreover, the amplitude of the flux linkage with the d-axis current is bigger than the $0 \mathrm{~A}$ current case.

This implies that the $\mathrm{d}$-axis current is powered in the direction to demagnetize the permanent magnets, lowering the total level of saturation, as shown in Fig. 7, which leads to the increase in the flux linkage of the permanent magnets. Thus, it is shown that the back-EMF due to the permanent magnets is increased when applying the d-axis current.

From the above results, it is confirmed that the magnitude, 


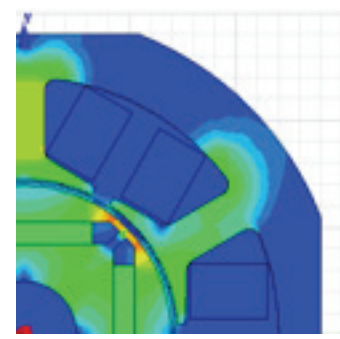

(a)

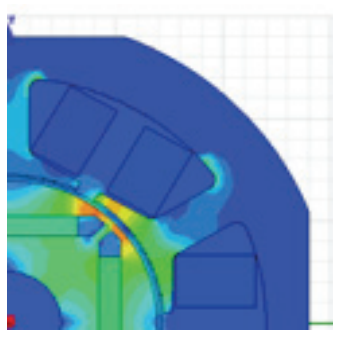

(b)

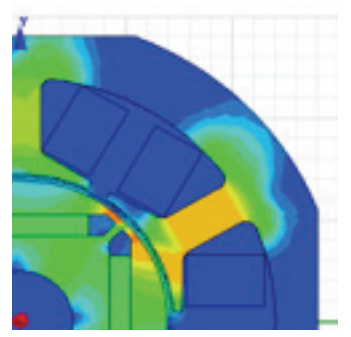

(c)

Fig. 7. Magnetic flux density distribution

harmonic component, and phase angle of the flux linkage can change based on the current amplitude. These points are factors when equivalent circuit simulation can consider.

Fig. 7 shows the magnetic flux density distribution when the current flows according to the previous three conditions. The magnetic flux density (Fig. 7(a)) caused only by the permanent magnets is greater than the flux density with the d-axis current (Fig. 7(b)), and the magnetic flux density (Fig. 7(c)) with the q-axis current becomes asymmetric. In other words, since the saturation of the permanent magnets becomes asymmetric, the back-EMF contains more harmonic components because of the combination of the permanent magnet $\mathrm{d}$-axis component and the q-axis component.

\subsection{Flux linkage on the rotating coordinate system by the d-axis current}

FOC is the control method transforming 3-phase variables on the dq-axis. Thus, all the characteristics should be taken into consideration after transforming the flux linkages on three-phase system to the dq-axis system.

When applying the d-axis current, the flux linkages of only the permanent magnets, of only the current, and of both the permanent magnets and current are decomposed into d-axis component and q-axis component in Fig. 8, and then the flux linkages are compared to the flux linkages of only the permanent magnets without the load current. The q-axis flux linkage component is, on average, close to zero, which implies it has only the d-axis component. The flux linkage of the d-axis current (Flux d only D) is negative and has the direction to demagnetize the permanent magnets. Thus, the magnitude of the flux linkage of the permanent magnets (flux d PM at D) gets to be greater than

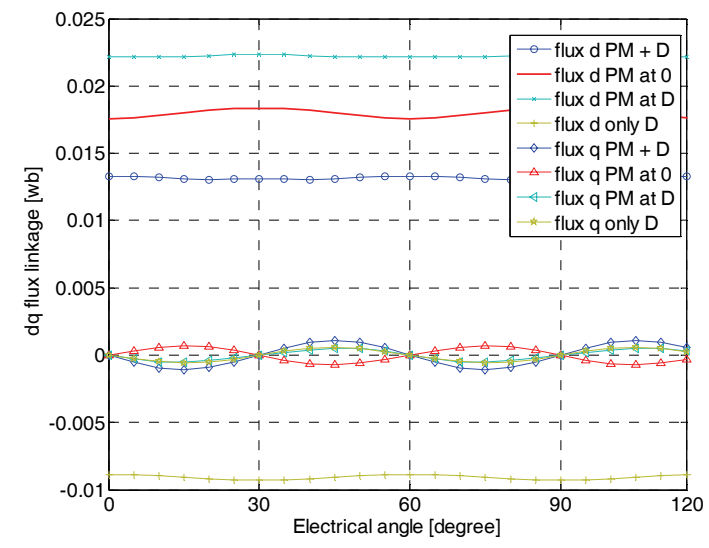

Fig. 8. Flux linkages by d-axis current

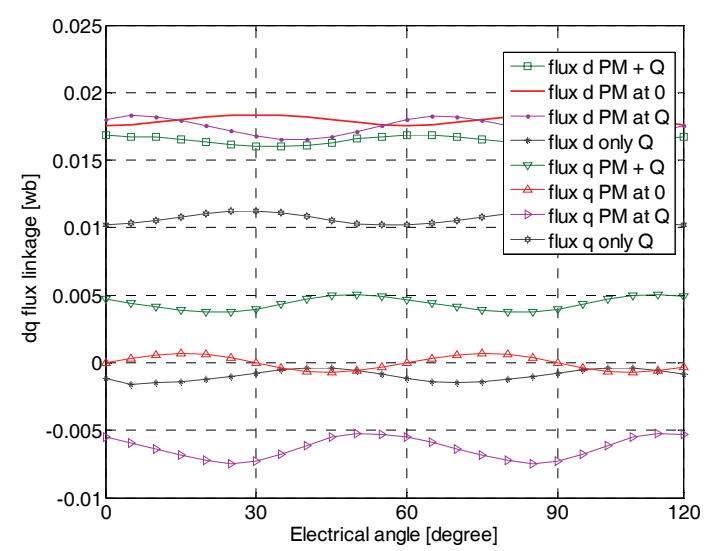

Fig. 9. Flux linkages by q-axis current

that of the flux linkage without the load current (flux d PM at 0 ). Therefore, it predicts that the torque calculation will have larger error under field weakening control conditions.

\subsection{Flux linkage on the rotating coordinate system for the q-axis current}

When current flows on the q-axis, the flux linkages of only the permanent magnets (PM at Q), of only the q-axis current (only Q), and of both the permanent magnets and qaxis current $(\mathrm{PM}+\mathrm{Q})$ are shown in Fig. 9.

The flux linkage of the permanent magnets and the qaxis current is decomposed into the d-axis component (flux d) and q-axis component (flux q). These cases are compared to the flux linkage of only the permanent magnets at no current. This shows that a flux linkage component appears on the d-axis and the q-axis due to the nonlinearity of saturation caused by the combination of the flux linkages of the permanent magnets on the d-axis and of the current on the q-axis. That is, the flux linkage component of the q-axis current (flux d only Q) is detected with the negative value on the d-axis partially, and the flux linkage component of the permanent magnets is partly observed with the negative value on the q-axis. Thus, these should be considered in calculating the torque. 


\section{Inductance Calculation}

\subsection{Phase inductance calculation}

As shown in the Eq. (1), the torque is directly affected by the difference between $\mathrm{L}_{\mathrm{d}}$ and $\mathrm{L}_{\mathrm{q}}$. For FOC, this is also used as the average value along with the case of back-EMF. However, it is widely known that the dq-inductance reduces as the current increases in IPM motors, due to the iron saturation depicted in Fig. 10.

Since the inductance changes more complexly because of the saliency in IPM motors, FEA is necessary to calculate inductance more accurately.

Fig. 11 illustrates the calculated A-phase inductance using FEA when the q-axis current is applied from 0 to $150 \%$. As the current increases, the inductance decreases because of the iron saturation, and the inductance waveforms are asymmetric because the inductance values are not sinusoidal due to the partial saturation of the iron. FFT analysis of this result is shown in Fig. 12. It shows that 2 nd harmonic and 6th harmonic are large. The 4th harmonic becomes the 6th harmonic component in dqreference frame.

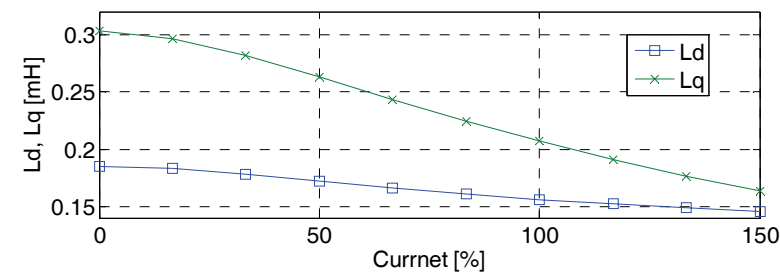

Fig. 10. Ld and Lq waveforms according to current variation

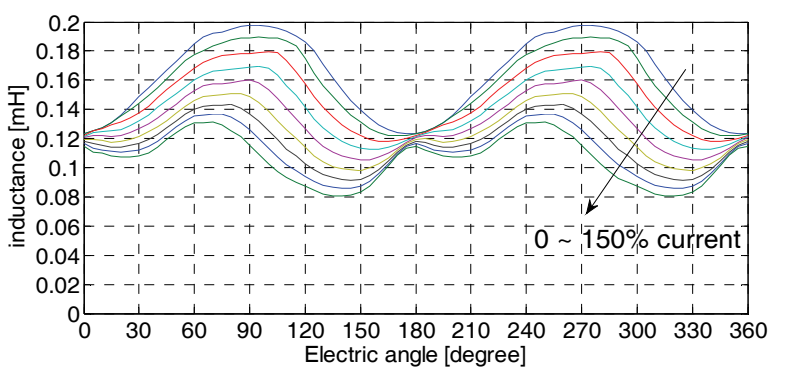

Fig. 11. Phase inductance waveforms according to the current variation

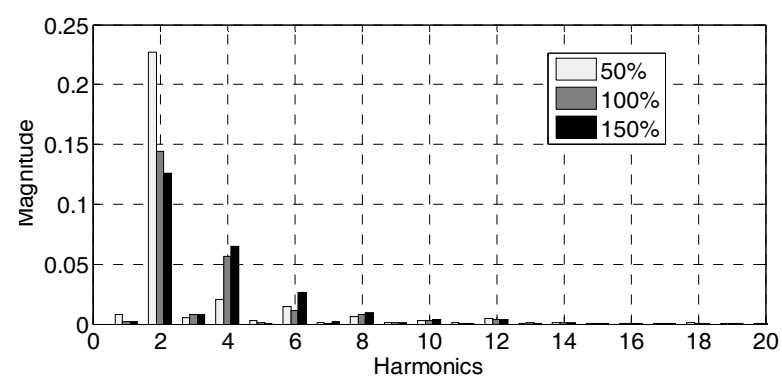

Fig. 12. Phase inductance FFT analysis according to the current variation
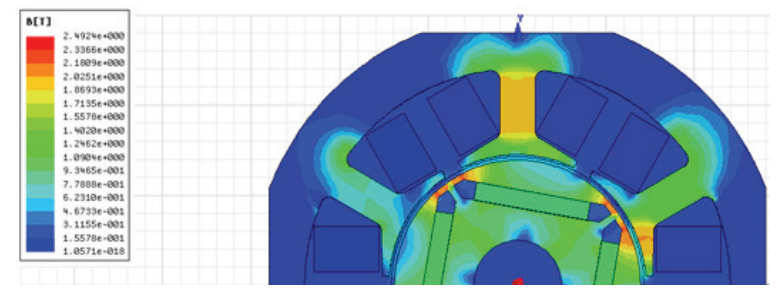

(a) 160 degree
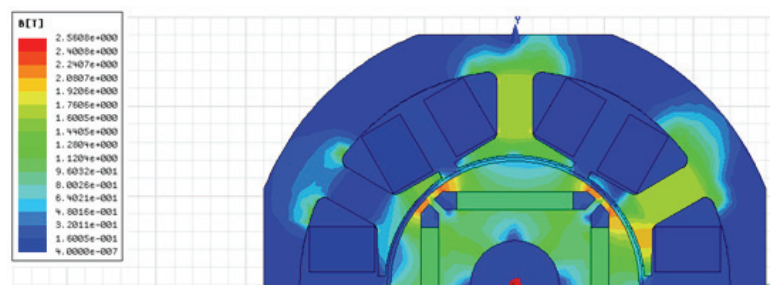

(b) 180 degree

Fig. 13. Flux density distribution

Fig. 13 describes the asymmetry of the phase inductance. Fig. 13(a) shows the flux density distribution at 160 electrical degrees, and Fig. 13(b) indicates the flux density distribution at 180 electrical degrees. The saturation is more immense at 160 electrical degrees rather than at 180 electrical degrees, and the flux is asymmetric due to the qaxis current component in Fig. 13(b).

Moreover, the inductance includes the harmonic components because the permanent magnet is located in the rotor of IPM motors, the distance from the magnet to the center of the motor is not constant, and the ribs on the edge of the permanent magnet are thin, which makes the saturation level uneven.

\section{$3.2 \mathrm{dq}$-axis inductance calculation}

As mentioned before, the asymmetric saturation of phase inductance is caused by the q-axis current, and this causes harmonic and asymmetric characteristics. In this case, it is expected that harmonic component and phase shift will be changed after dq-transformation is applied.

Fig. 14 shows the d-axis inductance when the q-axis current varied from 0 to $150 \%$ of rated current and Fig. 15 depicts the q-axis inductance. The angles of the q-axis inductance peak value vary, especially, when the current increases. This is expected to have an impact on the torque calculation. If the phase angle variation is also considered, a more precise interpretation could be possible.

Fig. 16 indicates the d-axis and q-axis inductances when the $\mathrm{q}$-axis current is varied from 50 to $150 \% . \mathrm{L}_{\mathrm{d}}-\mathrm{L}_{\mathrm{q}}$ at $150 \%$ current tends to be significantly lower than $\mathrm{L}_{\mathrm{d}}-\mathrm{L}_{\mathrm{q}}$ at $50 \%$ current, since the iron saturation leads to overall decrease of inductances. Thus, it is expected that the torque magnitude caused by reluctance difference in the torque component will be changed severely. Furthermore, the inductance varies periodically according to the angle 
because of the saliency of IPM motors, and the reason for the slight deviation of the waveform from the symmetry is the saturation effect by the q-axis current. Such an

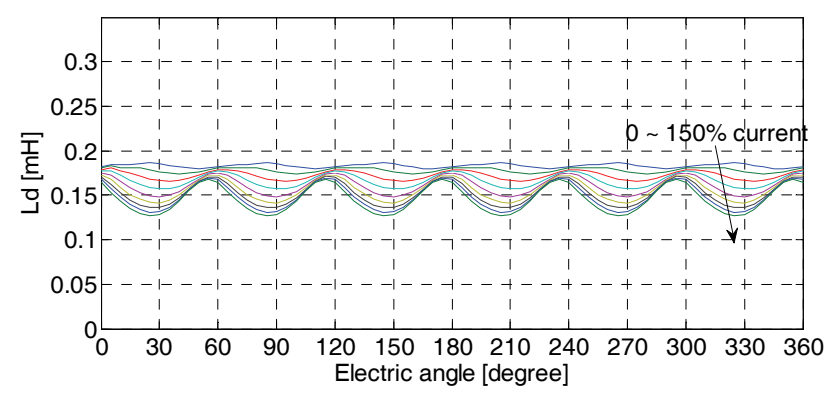

Fig. 14. $\mathrm{L}_{\mathrm{d}}$ according to various currents

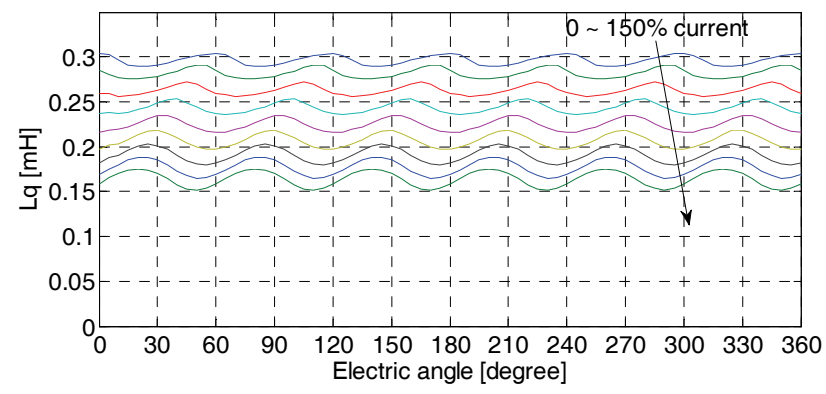

Fig. 15. $\mathrm{L}_{\mathrm{q}}$ according to various currents

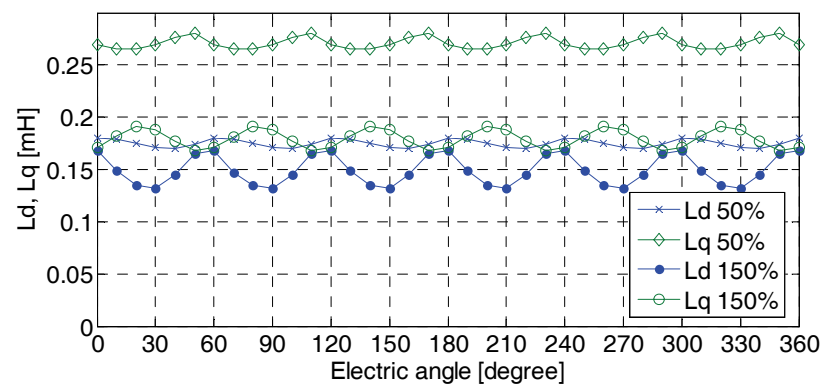

Fig. 16. $L_{d}$ and $L_{q}$ waveforms with $50 \%$ and $150 \%$ currents

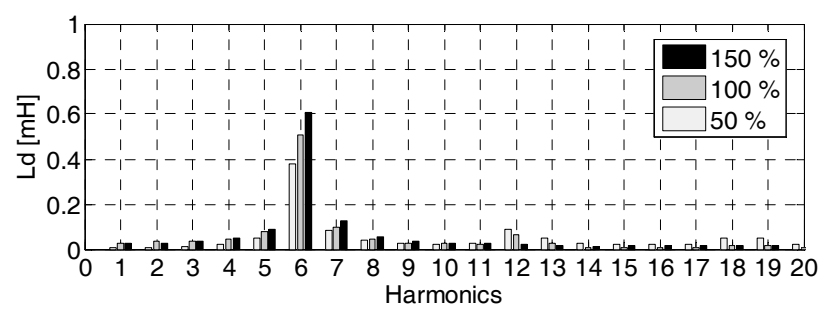

Fig. 17. $L_{d}$ FFT Analysis

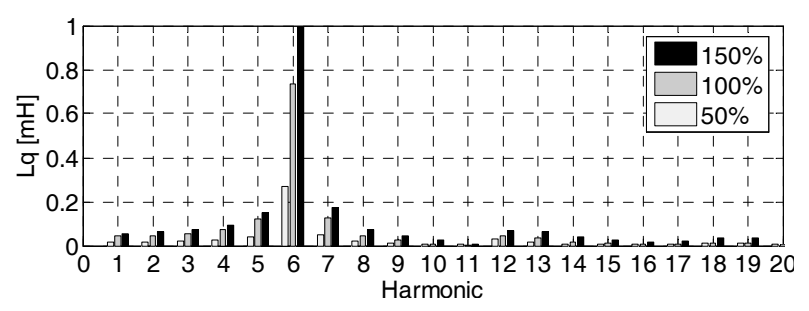

Fig. 18. $\mathrm{L}_{\mathrm{q}}$ FFT Analysis asymmetrical aspect implies that the torque characteristics will be asymmetric too.

Fig. 17 shows the d-axis inductance FFT analysis, which has a high 6th harmonic component, and there is no large variation with the changes in current.

In Fig. 18, the q-axis inductance FFT analysis is presented, which reveals significant 6th harmonic components and a large variation with the current changes.

When the phase of the 6th harmonic is considered, the phase changes as the current varies. These phase differences have an impact on the torque calculation, and considering these differences is expected to result in a more precise torque calculation.

\section{Torque Results}

The torques calculated in Eq. (1) for FOC and simulated in FEA are compared.

The torque only from applying the q-axis current is equal to Eq. (2) when substituting $i_{d}=0$ into Eq. (1) and assuming $\psi_{\mathrm{d}}=\psi_{\mathrm{f}}$.

Fig. 19 illustrates the calculated torque waveforms from Eq. (2).

$$
T_{e}=\frac{m P}{2}\left[\Psi_{d} i_{q}\right]=\frac{m P}{2}\left[\Psi_{f} i_{q}\right]
$$

In Fig. 19, the graphs represent the calculated torques from Eq. (2) and the d-axis flux linkage of the permanent magnets and the q-axis rated current $(\mathrm{PM}+\mathrm{Q})$, of only the permanent magnets without load current (PM at 0 ), of only the permanent magnets with the rated q-axis current (PM at Q), and the torque simulated by FEA (by FEA). As shown in Fig. 9, the d-axis total flux linkage is the summation of the d-axis flux linkages of the permanent magnets and the armature reaction of the q-axis current. There is a difference in the flux linkage with and without the load current. Therefore, there are corresponding results depending on which of $\psi_{\mathrm{d}}$ or $\psi_{\mathrm{f}}$ is applied to the Eq. (2) in Fig. 19.

Finally, the calculated torque deriving from the total flux linkage is closely similar to the result from FEA. However, there are differences between these torques in terms of

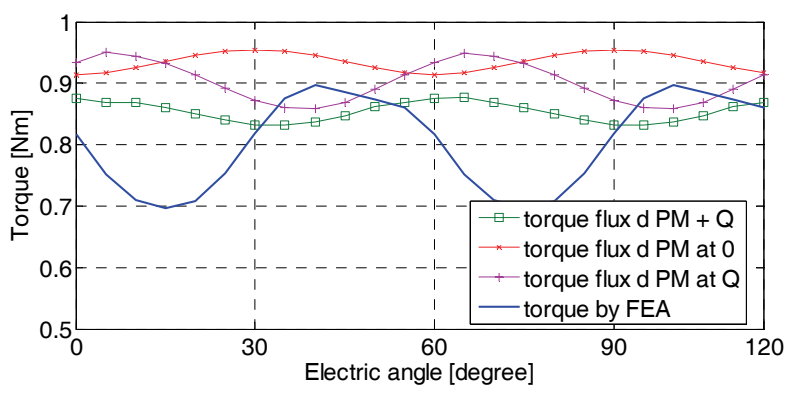

Fig. 19. Calculated torque waveforms 
the magnitude and phase because the reluctance torque component from $i_{q}$ current is omitted from the torque equation. Accordingly, it is clear that the torque from only the combination of $\psi_{\mathrm{f}}$ and $\mathrm{i}_{\mathrm{q}}$ can't be derived due to the armature reaction and saturation effects when applying the q-axis current.

\section{Conclusion}

Conventionally, the equivalent circuit for FOC assumes that the motor circuit parameters are sinusoidally distributed and linear, but for IPM motors these assumptions must be reconsidered because of the saliency and high saturation effect. This paper presents the nonlinear characteristics of the inductance and flux linkage from permanent magnets. It was shown that the cause of 6 th harmonic component was the inductance and back-EMF harmonics, the inductance and flux linkage were not symmetric due to the d-axis current, and d-axis and q-axis values were reviewed after dq-transformation. When applying current, the flux linkage of the permanent magnets changes due to the armature reaction and saturation. Particularly, FEA shows that the q-axis current generates the d-axis component. The flux linkage of only the permanent magnets was reviewed on $\mathrm{d}$-axis and q-axis, and it was shown that the q-axis current could generate the d-axis flux linkage too. These could be considered when calculating the torque, and FEA results proved that the d-axis flux linkage of only the permanent magnets when applying the d-axis current to demagnetize is larger than the flux linkage of only the permanent magnets without the load current. This can be used effectively when calculating the torque for field weakening control. $\mathrm{L}_{\mathrm{d}}$ and $\mathrm{L}_{\mathrm{q}}$ have harmonic components, and the magnitude as well as phase of the harmonic varies according to the current values. Considering these phase differences is expected to result in a more precise torque calculation.

For future work, more accurate torque prediction will be performed through vector control simulations and confirmed though experiments. This approach is expected to obtain reasonable results in real IPM Motor drives.

\section{Acknowledgements}

This research is financially supported by Changwon National University in 2013

\section{References}

[1] Rahman, K.M.; Hiti, S., "Identification of machine parameters of a synchronous motor," IEEE Transactions on Industry Applications, vol. 41, no. 2, pp. 557-565, March-April 2005

[2] Dutta, R.; Rahman, M.F., “A Comparative Analysis of Two Test Methods of Measuring d - and q -Axes Inductances of Interior Permanent-Magnet Machine," IEEE Transactions on Magnetics, vol. 42, no. 11, pp. 3712-3718, Nov. 2006

[3] Yoshiaki Kano ; Kyohei Watanabe ;Takashi Kosaka ; Nobuyuki Matsui, "A new modeling approach for circuit-field-coupled time-stepping electromagnetic analysis of saturated interior permanent magnet synchronous motors," EE. in Japan, Vol. 174, No. 1, pp. 49-58, 2011.

[4] O.A. Mohammed; S. Liu; Z. Liu, "Physical Modeling of PM Synchronous Motors for Integrated Coupling With Machine Drives," IEEE Transactions on Magnetics, vol. 41, no. 5, pp. 1628-1631, Nov. 2005

[5] Thomas Herold; David Franck; Enno Lange; Kay Hameyer, "Extension of a D-Q Model of a Permanent Magnet Excited Synchronous Machine by Including Saturation, Cross-Coupling and Slotting Effects," IEEE International Electric Machines \& Drive conference (IEMDC), 2011

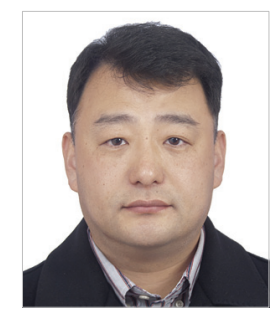

Kibong Jang He received Phd. degree in electrical engineering from Hanyang University. His research interests are analysis and drive of electric machines 